\title{
Light and Heavy Dark Matter Particles
}

\author{
C. Bøehm ${ }^{1}$, P. Fayet ${ }^{2}$, J. Silk ${ }^{1}$ \\ ${ }^{1}$ Denys Wilkinson Laboratory, 1 Keble Road, OX1 3RH, Oxford, England, UK \\ 2 Laboratoire de Physique Théorique de l'ENS, UMR 8549, 24 rue Lhomond, 75231 Paris Cedex 05, France
}

(10 November 2003)

\begin{abstract}
It has recently been pointed out that the $511 \mathrm{keV}$ emission line detected by Integral/SPI from the bulge of our galaxy could be explained by annihilations of light Dark Matter particles into $e^{+} e^{-}$. If such a signature is confirmed, then one might expect a conflict with the interpretation of very high energy gamma rays if they also turn out to be due to Dark Matter annihilations. Here, we propose a way to accomodate the existence of both signals being produced by Dark Matter annihilations through the existence of two stable (neutral) Dark Matter particles, as is possible in theories inspired from $N=2$ supersymmetry.
\end{abstract}

07.85.Fv, 52.38.Ph, 95.35.+d, 78.70.Bj, 14.80.-j

\section{INTRODUCTION}

Dark Matter particles are expected to produce indirect annihilation signatures via production of high energy $e^{+}, \bar{p}, \gamma, \nu$ which are potentially detectable [1]. An especially intriguing signal has recently been observed with the SPI spectrometer on the INTEGRAL (INTErnational Gamma-Ray Astrophysics Laboratory) satellite, which detected a bright $511 \mathrm{keV} \gamma$-ray line from the galactic bulge [2]. This detection turns out to be in good agreement with previous measurements [3] and fits reasonably well to a gaussian with full-width half-maximum of $\sim 9^{\circ}$, with a $2 \sigma$ confidence interval of $6^{\circ}-18^{\circ}$.

Positrons in the galactic bulge can be emitted by such astrophysical objects as for example hypernovae [4], neutron stars or black holes [5] etc. However, whether these sources can be at the origin of this $511 \mathrm{keV}$ line is still under debate $[6,7]$, especially in the bulge which consists exclusively of old, low mass stars. As an alternative explanation, annihilations of light Dark Matter particles $[8,9]$ into $e^{+} e^{-}$seem to provide a surprisingly good explanation for the Integral measurement, if the dark matter halo profile is approximated in the centre of the galaxy as $\rho(r) \propto r^{-\gamma}$ with $\gamma \in[0.4-0.8]$ [10]. Such a value corresponds to a mild cusp intermediate between the NFW distribution [11] and the profile extrapolated from observations of dwarf and low surface brightness galaxies [12]. This empirically-derived profile is consistent with a density profile that incorporates information on the dark matter contribution constrained by gravitational microlensing measurements of the Milky Way bulge [13]. Moreover the inferred annihilation cross section is concordant with that inferred from the relic density, without any need to assume a boost factor, due for example to dark matter clumpiness.

One way to determine whether the $511 \mathrm{keV}$ line is due to astrophysical sources or Dark Matter (DM) annihilations is to seek a similar signature from low surface brightness dwarf galaxies [14]. Those objects are everywhere dark matter-dominated. Therefore, if the emission line detected in our galaxy is due to DM annihilations, then one should also detect a $511 \mathrm{keV}$ line from nearby dwarf spheroidals. The flux from the Sagittarius Dwarf Galaxy, in particular, appears to fall within the expected sensitivity of Integral/SPI. It is therefore quite likely that, in the near future, Integral/SPI will be able to rule out or confirm the existence of Light Dark Matter (LDM) particles.

Other experiments seek to detect very high energy gamma rays (i.e. of order a hundred $\mathrm{GeV}$ ) [15]. Clearly, if the assumption of LDM were to be confirmed, it would seem difficult to accomodate low as well as possible high energy gamma ray signatures (unless, of course, astrophysical sources appear to be at the origin of the high energy gamma rays). In fact, the latter may have a natural explanation within the framework of the (Minimal) Supersymmetric Standard Model. Annihilations of the lightest neutralino are indeed expected to give rise to gamma rays of up to several hundred $\mathrm{GeV}$. Whether they would appear a satisfactory solution or not then depends on the angular and energy distribution of the observed gamma rays.

However, it is difficult to accomodate very large neutralino masses (as required if very energetic gamma rays are detected), with the large annihilation cross sections needed to comply with the sensitivity of present/next generation gamma ray experiments, unless one invokes the supermassive black hole at the centre of our galaxy to steepen the innermost dark matter cusp within the zone of influence of the black hole and thereby enhance the predicted annihilation rate [16]. The amplitude of this effect is of course dependent on uncertain astrophysical assumptions about the formation of the supermassive black hole.

Also, it is worth mentioning that the adoption of very large neutralino masses requires one to invoke the coannihilation mechanism in order to produce a low enough relic density. However, the conditions for coannihilation to operate are not always satisfied, as shown in [17]. In particular, the decay process of the particle with which the neutralino is supposed to coannihilate (namely the Next-to-Lightest Supersymmetric Particle, NLSP) may be more efficient than the processes that can regenerate this particle. As a result, one is left with the annihila- 
tion region (allowing for neutralino masses up to $\sim 100$ $\mathrm{GeV}$ ), plus potentially only a small region of the supersymmetric parameter space corresponding to the largest neutralino masses (i.e. for which the neutralino is almost mass degenerate with the NLSP and where the efficiency of the NLSP decay process is significantly reduced).

In this Letter, we point out that it is possible to reconcile the low and high energy signatures, even if both of them turn out to be due to Dark Matter annihilations. Indeed, existing theories predict two different kinds of Dark Matter particles [9]. For example, in a $N=1$ supersymmetric framework with mirror fermions (somehow reminiscent of $N=2$ extended Supersymmetry) [18], one expects the existence of two neutral and stable particles. One would be a heavy fermion for example, like the lightest neutralino, and the other one a possibly light spin-0 particle. Both of them would be neutral and also stable as a result of two discrete symmetries (say $R$ and $M$-parities).

The light particle would be crucial in order to achieve the correct relic density, while heavy stable particles would be at the origin of very high energy gamma rays. Light particles would be difficult to detect in direct Dark Matter detection experiments because of their mass but heavy particles would also be hard to find due to their small residual number densities.

\section{RELIC DENSITY, 511 KEV LINE AND HIGH ENERGY GAMMA RAYS}

Let us assume that there indeed exists two DM particles. The first question to deal with is: how do they share the observed relic density [19]?

If the $511 \mathrm{keV}$ emission line is indeed due to LDM annihilations in the centre of the Milky Way, then one needs a cross section (times the relative velocity, normalised to c) of about $\sim 10^{-4}$ to $10^{-5} \mathrm{pb}$ to fit the observed flux $\left(9.9 \times 10^{-4} \mathrm{ph} \mathrm{cm}^{-2} \mathrm{~s}^{-1}\right)$ and the angular distribution of $\gamma$-rays indicated by Integral/SPI.

On the other hand, it is also necessary to avoid an overproduction of relic LDM for the observed low energy gamma ray flux or an overproduction of gamma rays for a given relic LDM abundance. Since low DM masses actually increase the amount of annihilations in our galaxy, and consequently the flux of low energy gamma rays that has been well measured by several experiments, one has to invoke a $v^{2}$ suppressed annihilation cross section. One way to achieve this is to assume a scalar particle coupled to a new light gauge boson $[9]^{*}$.

\footnotetext{
*This gauge boson is similar to the one introduced in [20,21]. However, in the case we discuss here, it would be coupled to Dark Matter and, in fact, would mainly decay into two DM particles.
}

The LDM cross section would then be much larger at early times than is expected in our galaxy [8]. For example, one expects their annihilation cross section to be about $10^{-5} \mathrm{pb}$ in the Milky Way, to explain the $511 \mathrm{keV}$ line, and about $1 \mathrm{pb}$ in the primordial Universe (since the DM velocity is $\sim 310^{-3}$ the velocity in the primordial Universe).

Given the relationship between the relic density of annihilating DM particles and the annihilation cross section in the primordial Universe, the cosmological parameter associated with these light particles is then expected to be about

$$
\Omega_{\mathrm{dm}}^{t h} h^{2} \simeq 0.024 \times \frac{x_{F}}{\sqrt{g_{\star}}} \times\left(\frac{\langle\sigma v\rangle_{p}}{1 \mathrm{pb}}\right)^{-1}
$$

where $x_{F}=m_{\mathrm{dm}} / T_{F} \simeq 12-19$ for particles in the MeV$\mathrm{GeV}$ range, $g$ and $g_{\star}$ are the numbers of internal and relativistic degrees of freedom, respectively and $v$ is expressed in units of $c$. Note that $\langle\sigma v\rangle_{p}$ stands for the primordial annihilation cross section of LDM.

For $\sim \mathrm{MeV}$ particles, the value of $\frac{x_{F}}{\sqrt{g_{\star}}}$ is $\sim 4$. Thus one obtains $\Omega_{\mathrm{dm}}^{t h} h^{2} \simeq 0.095\left(\langle\sigma v\rangle_{p} /(1 \mathrm{pb})\right)^{-1}$. This has to be compared to $\Omega_{\mathrm{dm}} h^{2} \sim 0.1$ as found by WMAP.

LDM particles are therefore likely to fit the observed relic density, leaving little room for another "weakly annihilating" species. Of course, this depends on the profile used and the exact cross section needed to fit the angular resolution of the SPI detection. If the annihilation cross section of heavy DM particles $\left(\langle\sigma v\rangle^{\prime}\right)$ is larger than $1 \mathrm{pb}$ at both early epochs and today ${ }^{\dagger}$, then these particles are likely to be subdominant but, on the other hand, they would yield significant gamma ray fluxes! This picture would then lead to a situation where one can fit the observed relic density, explain the nature of the $511 \mathrm{keV}$ line if it is confirmed and potentially predict, at the same time, a non-negligible flux for high energy gamma rays, even if the dark halo profile is not as cuspy as the NFW profile.

Assuming that LDM fulfills the relic density condition, one can study the effect of a large annihilation cross section associated with heavy particles. Of course, the larger this cross section gets, the less credible it becomes (as one expects $\sigma \propto 1 / m_{\chi}^{2}$ or $\propto 1 / m_{S}^{2}$, where $\chi$ and $S$ denote the heavy DM particle or the exchanged particle respectively). However, it is worth remembering that resonances are possible, in which case they would significantly enhance the cross section.

The $511 \mathrm{keV}$ emission line seems to favor a quite flat profile (for example $\gamma \sim 0.4$ ). If so, then the annihilation cross section needed may appear extremely large. In fact, this would be probably too large compared to what could

\footnotetext{
${ }^{\dagger}$ One would then require constant annihilation cross sections for heavy particles.
} 
be realistically expected in a particle physics model unless, perhaps, new kinds of interactions are invoked. On the other hand, even a quite mild dark halo profile (i.e. $\gamma \sim 0.8$ ) would yield a significant amount of high energy gamma rays if the annihilation cross section associated with heavy DM particles turns out to be large but still "realistic" (i.e. enhanced by resonance effects for example). Such a scenario would then be in agreement with the Dark Matter cosmological parameters measured by WMAP as well as with low and high energy annihilation signatures (if they were both confirmed).

\section{A THEORY WITH TWO STABLE DARK MATTER PARTICLES}

A very important requirement regarding any Dark Matter candidate is its stability. In a Standard Supersymmetric framework, the neutralino (absolute) stability is obtained through a $Z_{2}$ discrete symmetry, called $R$ parity [22]. This symmetry implies the decay of a supersymmetric particle into another one, plus standard model particles. This is what prevents the lightest supersymmetric particle (LSP) from decaying.

Since we are now looking for two stable particles, it is particularly interesting to start working within the framework of $N=2$ extended supersymmetric theories, as they may naturally allow for two $Z_{2}$ discrete symmetries, and may ultimately lead to two new distinct DM candidates. We shall denote these two discrete symmetries as $R$ and $M$ parities.

Note that the particles which matter for our purpose (say mirror leptons/quarks and new spin-0 states that couple these mirror particles to ordinary ones [18]) may be kept even after one abandons the full $N=2$ supersymmetry in favor of a $N=1$ theory.

\section{A. Two discrete symmetries}

As mentioned before, $N=1$ supersymmetric theory may admit a discrete $Z_{2}$ symmetry. The latter can be seen, in fact, as a remnant of a continuous $R$-symmetry $\left(R_{p}=(-1)^{R}\right)$.

Thus, quite similarly to the case $N=1$, an extended $N=2$ theory, for which there are now two supersymmetry generators

$$
Q=\left(\begin{array}{c}
Q_{L}^{1} \\
Q_{L}^{2}
\end{array}\right)
$$

may admit a $S U(2) \times U(1)$ global $R$-symmetry, that acts on the doublet $Q$.

Such a global $R$-symmetry allows one to act independently on the two supersymmetry generators $Q_{1}$ and $Q_{2}$. One can therefore define two distinct $R$-parity symmetry operators, $R_{1 p}$ and $R_{2 p}$; the usual $R$-parity symmetry corresponding to the product

$$
R_{p}=R_{1 p} R_{2 p} .
$$

By abandoning the full $N=2$ supersymmetry, one singles out one of the two supersymmetry generators (say $Q_{1}$ ) and redefines one of the two discrete symmetries (say $\left.R_{2 p}\right)$ as the $M$-parity symmetry.

We are then left with two discrete symmetries: the $M$-parity that can be seen as a $Z_{2}$ remnant of a continuous $U(1)$ symmetry $(M)$ acting globally on $N=1$ superfields, and the "standard" $R$-parity. Both of them are kept intact after spontaneous breaking of the electroweak symmetry.

\section{B. Particle spectrum from extended supersymmetry}

We now have to specify what is the expected spectrum from extended supersymmetry and, more precisely, which particle could be the LMP.

When $N=2$ extended supersymmetric theories are formulated in terms of $N=1$ superfields, ordinary gauge superfields are accompanied by additional $(N=1)$ "chiral gauge superfields". Together, they describe $N=2$ massless gauge multiplets containing, for example, two gluino octets, two photinos, etc., as well as two coloroctets of spin- 0 gluons, two spin-0 photons, etc. [18]. (These new "scalar gauge fields" may also appear as originating from the fifth and sixth components of higherdimensional gauge fields $V^{\hat{\mu}}$, in a six-dimensional spacetime [23].)

Matter (and also Higgs) chiral superfields systematically occur in pairs so as to describe $N=2$ "hypermultiplets". Since quark and lepton fields belong to lefthanded electroweak doublets and right-handed singlets, one has to introduce mirror particles which belong to right-handed doublets and left-handed singlets. This also necessitates however the introduction of appropriate symmetry breaking mechanisms, allowing one to reduce the full $N=2$ supersymmetry down to $N=1$.

Mirror quarks and leptons $\left(q_{M}\right.$ and $\left.l_{M}\right)$ may then acquire large masses through large Yukawa couplings with electroweak Higgs doublets (namely $H_{1}$ and $H_{2}$ in the standard supersymmetry framework). They would have evaded past accelerator searches if heavier than a few hundred GeV's, but may still show up at LHC.

The breaking of the $(N=2)$ supersymmetry may be elegantly obtained by demanding periodic and antiperiodic boundary conditions for ordinary $R$-even particles and their $R$-odd superpartners, respectively - in which case the masses of the (lowest-lying) gravitinos, gluinos and photinos, which fix the energy scale at which supersymmetric particles should start to show up, would be given, in the simplest case and up to radiative correction effects, by $m_{3 / 2}=m_{1 / 2}=\frac{\pi \hbar}{L c}=\frac{\hbar}{2 R c} . L$ is the size of the extra dimension responsible for supersymmetry breaking. This led us to consider the possibility of relatively "large" extra dimensions, associated with a 
compactification scale that could then be as "low" as $\mathrm{TeV}$ scale [24].

It is quite conceivable that the new spin- 0 states, as well as mirror lepton and quark fields, may only manifest themselves at the compactification scale. However, whether the extra spin- 0 components of gauge fields actually show up or not in the low-energy theory depends on the details of the mechanism that should be responsible for the breaking of the extended supersymmetry (and/or the compactification of the extra space dimensions). Since this mechanism is unknown, one can still discuss the possibility that they appear in the low-energy theory, much below the compactification scale.

The new spin- 0 bosons - originally appearing as extra degrees of freedom for spin-1 gauge fields - will then have Yukawa couplings relating quarks and leptons to their mirror partners. They should lead to new decay modes ( $M$-parity invariant) like

$$
q_{M}\left(\text { or } l_{M}\right) \rightarrow q(\text { or } l)+\text { neutral spin-0 particle . }
$$

Note that $M$-parity is equal to +1 for ordinary and Supersymmetric Standard Model particles (including those described by the doublet Higgs superfields $H_{1}$ and $H_{2}$ ). It is equal to -1 , on the other hand, for mirrors, their superpartners, the new spin-0 particles, the new inos (second octet of gluinos, additional charginos and neutralinos) and finally also for the extra Higgs bosons which appear as the trace of the underlying extended supersymmetry.

The lightest of the new $M$-odd particles, that remains at the end of such decay chains, is then expected to be stable.

\section{A Light Dark Matter candidate}

In the scenario described above, one ends up with a new neutral and stable ( $M$-odd) particle that can play the rôle of LDM candidate.

This particle could be a scalar (that couples ordinary to mirror particles). In which case, it is expected to annihilate in pairs through mirror fermion exchanges, as discussed in [9]. However, such a scenario is disfavored by the $511 \mathrm{keV}$ line observation (which requires $v^{2}$ suppressed annihilation cross section), unless the associated cross section turns out to be extremely small in the primordial universe (namely $\lesssim 10^{-31} \mathrm{~cm}^{3} \mathrm{~s}^{-1}$, which requires heavy mirror fermions and probably chiral couplings).

The LDM particle may also annihilate through possible couplings to a spin-1 $U$ boson, if the extra $U(1)$ symmetry generator includes a contribution involving the continuous $U(1) M$-symmetry generator ${ }^{\ddagger}$. In this case, one

\footnotetext{
${ }^{\ddagger}$ Such a gauging of an extra $U(1)$ not commuting with the
}

would obtain an attractive LDM candidate that could explain the low energy signature without being in conflict with a signal at higher energies.

\section{SUMMARY}

Supersymmetric theories originating from $N=2$ have the pleasant feature of providing two discrete symmetries, that we denote $R$ and $M$-parities. When they are conserved, one expects that both the LSP (Lightest Particle invariant under $R$-parity) and LMP (Lightest Particle of the spectrum invariant under $M$-parity) become absolutely stable. Such a scenario would then offer two DM candidates: one would be the $R$-odd LSP and the other one, the $M$-odd LMP.

Among the new $M$-odd particles, one finds spin-0 states. The case where one of them turns out to be the lightest of the $M$-odd spectrum appears particularly appealing as it could play the rôle of Light Dark Matter candidate (with a mass of a few MeV-100 MeV). Provided one introduces a new light gauge boson $U$, one could evade low energy gamma ray constraints [9] and also explain the $511 \mathrm{keV}$ emission line (if it is seen to be due to DM annihilations) $\S$.

To avoid an unwanted production in $Z$ decays, this spin-0 particle should have no or negligible direct couplings to the $Z$. This would restrict potential LMP candidates to e.g. a spin-0 photon, or companion of the weak hypercharge gauge field $B^{\mu}$.

In such a scenario, one would for example end up with the "standard" (lightest) neutralino as the $R$-odd LSP and a new neutral spin-0 particle, as $M$-odd LMP.

The new $M$-odd spin-0 states are expected to couple quarks and leptons to mirror partners. However, if the latter are very heavy (a few hundred GeV for example), one does not expect a significant contribution to low energy gamma rays. (The associated annihilation cross section would indeed be dominated by a constant term but this one is not expected to yield any significant contribution as it would be lower than $10^{-5} \mathrm{pb}$. ) On the other hand, one expects high energy gamma rays from the annihilations of heavy DM particles for which $\sigma v^{\prime}>1 \mathrm{pb}$.

This scenario finally evades several astrophysical and particle physics constraints including too large a contribution to the muon and electron $g-2$. On the other hand, a gauge boson is expected to bring an extra contribution to the $\nu-e$ elastic scattering cross section at

$N=2$ supersymmetry may however generate anomalies (at least superficially).

${ }^{\S}$ There is no need to introduce a gauge boson for $\sim 100 \mathrm{MeV}$ but this case appears borderline regarding the $511 \mathrm{keV}$ line, the relic density criterion and the nucleosynthesis constraint. 
low energy. The latter has been measured by two experiments (LAMPF and LSND). No significant deviations have been found $[25,26]$. Therefore, it is necessary to either impose a dissymmetry between the coupling of the new gauge boson to DM and its coupling to standard model particles or, alternatively, to suppress its coupling to neutrinos (e.g. by having the $q$ and $l$ contribution to the $U$ current proportional to the electromagnetic current, as discussed in [21]).

Of course, there may exist other ways out to achieve a $v^{2}$ suppressed cross section for LDM. We do not discuss them in this letter.

By having two kinds of DM particles, we then fulfill the correct relic density, potentially explain the $511 \mathrm{keV}$ emission line detected by Integral/SPI and simultaneously predict high energy gamma rays.

This would turn out to be particularly interesting either if the smoking gun proposed in [14] reveals to be in favor of LDM and if high energy diffuse gamma rays were identified as coming from Dark Matter annihilations, or simply if the high energy gamma rays turned out to be associated with a Dark Matter annihilation cross section that is much larger compared to what is needed to achieve the correct relic density.

[1] J. Silk and M. Srednicki, Phys. Rev. Lett. 53, 624 (1984).

[2] J. Knodlseder, et.al., Accepted for publication in A\&A, arXiv:astro-ph/0309442; P. Jean et al., arXiv:astro$\mathrm{ph} / 0309484$.

[3] D. D. Dixon et al., arXiv:astro-ph/9703042; P. A. Milne, J. D. Kurfess, R. L. Kinzer and M. D. Leising, arXiv:astro-ph/0106157.

[4] S. E. Woosley and A. Heger, arXiv:astro-ph/0309165.

[5] R. E. Lingenfelter and R. Ramaty, Positron-Electron Pairs in Astrophysics, eds. M. L. Burns, A. K. Harding and R. Ramaty, AIP Conference Proceedings, 267.

[6] P. A. Milne, L. S. The and M. D. Leising, arXiv:astroph/0104185. P. A. Milne, J. D. Kurfess, R. L. Kinzer and M. D. Leising, New Astron. Rev. 46, 553 (2002) and references therein.

[7] M. Pohl, arXiv:astro-ph/9807268.

[8] C. Boehm, T. A. Ensslin and J. Silk, arXiv:astro$\mathrm{ph} / 0208458$.

[9] C. Boehm and P. Fayet, arXiv:hep-ph/0305261.

[10] C. Boehm et al., arXiv:astro-ph/0309686.

[11] J. F. Navarro, C. S. Frenk and S. D. White, Astrophys. J. 490, 493 (1997).

[12] A. V. Kravtsov, A. A. Klypin, J. S. Bullock and J. R. Primack, arXiv:astro-ph/9708176.

[13] J.J. Binney, N.W. Evans, astro-ph/0108505.

[14] D. Hooper et al, astro-ph/0311150.

[15] A. Djannati-Atai, for the H.E.S.S. collaboration "First results from southern hemisphere extragalactic observations with H.E.S.S.", Presentation at ICRC 2003,
Tsukuba; R. Enomoto (ICRR), S. Hara (TIT), CANGAROO collaboration, Astropart.Phys. 16 (2002) 235-244.

[16] G. Bertone, G. Sigl and J. Silk, Mon.Not.Roy.Astron.Soc. 337 (2002) 98.

[17] C. Boehm, D. Hooper and J. Silk, in preparation.

[18] P. Fayet, Nucl. Phys. B149, 137 (1979); Proc. 1980 Karpacz School (Harwood, 1981) p.115; Phys. Lett. B142, 263 (1984); Nucl. Phys. B246, 89 (1984).

[19] C. L. Bennett et al., "First Year Wilkinson Microwave Anisotropy Probe (WMAP)", arXiv:astro-ph/0302207.

[20] P. Fayet, Phys. Lett. B95, 285 (1980); Nucl. Phys. B187, 184 (1981).

[21] P. Fayet, Nucl. Phys. B347, 743 (1990).

[22] P. Fayet, Phys. Lett. B69, 489 (1977); G. Farrar and P. Fayet, Phys. Lett. B76, 575 (1978).

[23] P. Fayet, Phys. Lett. B159, 121 (1985).

[24] P. Fayet, Nucl. Phys. B263, 649 (1986); Proc. 2nd Nobel Symp. on El. Part. Physics (Marstrand, Sweden, 1986), Physica Scripta T 15 (1987) 46.

[25] R. C. Allen et al., Phys. Rev. D47, 11 (1993).

[26] LSND coll., Phys. Rev. D63, 112001 (2001). 